Obere Extremität 2021 · 16:68-74 https://doi.org/10.1007/s11678-020-00564-2 Received: 3 October 2019

Accepted: 26 February 2020

Published online: 3 April 2020

(c) The Author(s) 2020

\author{
Marc Schnetzke ${ }^{1,3} \cdot$ Susan Sulzer ${ }^{2}$ Jennifer Engelke ${ }^{1}$ Markus Loew ${ }^{3}$ \\ 'BG Trauma Center Ludwigshafen, University of Heidelberg, Ludwigshafen, Germany \\ ${ }^{2}$ Complejo Hospitalario San José, Santiago, Chile \\ ${ }^{3}$ ATOS Clinic Heidelberg, Heidelberg, Germany
}

\title{
Cemented all-polyethylene glenoid with standard or individualized backside curvature
}

\section{A retrospective comparative study}

\begin{abstract}
Anatomic shoulder replacement is a safe and effective therapy for the treatment of primary glenohumeral osteoarthritis and other shoulder joint disorders $[8,13$, $28,33,34]$. A standard-stem prosthesis in combination with an all-polyethylene glenoid currently represents the gold standard of anatomic shoulder replacement $[10,16,23]$. Revision of standardstem prostheses is challenging and frequently associated with relevant bone loss from the proximal humerus $[1,4]$. De-

The aim of the current study was to compare the clinical and radiographic results of this new all-polyethylene glenoid with variable backside curvature adapted to the individual anatomy with a standard all-polyethylene glenoid without variable curvature. The hypothesis was that the new all-polyethylene glenoid with variable backside curvature adapted to the individual anatomy has a significantly lower rate of radiolucent lines on followup 2-4 years postoperatively.
\end{abstract} velopments in recent years have therefore focused primarily on the humeral portion of shoulder prostheses and have resulted in the introduction of the fourth generation of shoulder prostheses with stemless and short-stem prostheses with metaphyseal anchoring [13, 29-31].

The weak link in anatomic shoulder replacement, however, is the glenoid component $[14,26,41]$. Loosening rates of $30-70 \%$ in long-term follow-up of more than 10 years are described in the literature $[7,38]$. The focus of more recent developments has thus been on optimizing the design of the glenoid component with individualized adaptation to the glenoid anatomy. Raiss et al. recently published the 2-year results of an all-polyethylene glenoid with variable backside curvature adapted to the individual anatomy [27]. The authors reported that the short-term results are promising with no loosening of the glenoid component and a low rate of radiolucent lines.

\section{Materials and methods}

All consecutive patients who received an anatomic shoulder replacement from the senior author (ML) from October 2010 to July 2016 were included in this retrospective comparative single/center study. Inclusion criteria were:

1. The indication of primary glenohumeral osteoarthritis

2. Anatomic shoulder replacement with a short-stem prosthesis (Ascend ${ }^{\mathrm{m}}$, Wright Medical, Memphis, TN, USA) and a keeled all-polyethylene glenoid

3. Follow-up period of at least 24 months

The exclusion criteria were:

1. Existing damage to the rotator cuff

2. Previous infection in the affected shoulder joint
Following a review of the inclusion and exclusion criteria, 92 patients with 99 implanted shoulder prostheses were included. In the context of this study, 87 of 99 shoulder joints (follow-up rate: $88 \%$ ) could be examined. Three patients refused to participate, one patient died from unrelated reasons, and eight patients were unable to participate in the follow-up because of health problems. Of the 87 shoulders, 42 (48\%) underwent arthroplasty with a standard all-polyethylene glenoid (group 1; Ascend Aequalis Glenoid, Wright Medical, Memphis, TN, USA) from October 2010 to June 2013 , and $45(52 \%)$ with an all-polyethylene glenoid with variable backside curvature adapted to the individual anatomy (group 2; Ascend ${ }^{\text {m }}$ Perform Glenoid, Wright Medical) from July 2013 to July 2016.

The mean age of all patients at the time of surgery was 68 years (range, 34-84); 38 patients (44\%) were male and $49(56 \%)$ were female. In 48 cases (55\%) the right shoulder was operated on and in 39 cases (45\%) the left shoulder. The two groups were comparable with respect to epidemiological parameters (• Table 1; $p \geq 0.251$ ).

\section{Operative technique}

All procedures were carried out by the senior author of this study (ML) using a standardized technique. Subscapularis 


\begin{tabular}{|c|c|c|c|}
\hline & Aequalis $(n=42)$ & Perform $(n=45)$ & $p$ \\
\hline \multicolumn{4}{|l|}{$\operatorname{Sex}(n ; \%)$} \\
\hline Male & $21(50 \%)$ & $17(38 \%)$ & \multirow[t]{2}{*}{$0.251^{\mathrm{a}}$} \\
\hline Female & $21(50 \%)$ & $28(62 \%)$ & \\
\hline $\begin{array}{l}\text { Age at } \\
\text { surgery (mean } \pm S D \text {; } \\
\text { range) }\end{array}$ & $67 \pm 8(52-82)$ & $68 \pm 10(34-84)$ & $0.577^{\mathrm{b}}$ \\
\hline \multicolumn{4}{|l|}{ Side of surgery $(n ; \%)$} \\
\hline Right & $23(55 \%)$ & 25 (56\%) & \multirow[t]{2}{*}{$0.941^{\mathrm{a}}$} \\
\hline Left & $19(45 \%)$ & $20(44 \%)$ & \\
\hline Dominant side & $23(55 \%)$ & $25(56 \%)$ & $0.941^{\mathrm{a}}$ \\
\hline $\begin{array}{l}{ }^{\mathrm{a} C} \text { Chi-square test } \\
{ }^{\mathrm{b}} \boldsymbol{t} \text { Test }\end{array}$ & & & \\
\hline
\end{tabular}

tenotomy was performed via a deltopectoral approach. Tenodesis of the long head of the biceps was undertaken as standard. Following exposure, the humeral head was resected at the level of the anatomic neck either by a free-hand technique or using a resection guide depending on the intraoperative decision of the surgeon. The glenoid was then exposed. Glenoid size was determined by means of the sizer present. Three different sizes (small, medium, large) were available in group 1 with a standard backside curvature. In group 2 an "extra-large" size was also available. Additionally, in group 2, the radius of curvature of the glenoid was determined (sizes small and medium: $30 \mathrm{~mm}$, $35 \mathrm{~mm}$, and $40 \mathrm{~mm}$; sizes large and extra-large: $40 \mathrm{~mm}, 50 \mathrm{~mm}$, and $60 \mathrm{~mm}$ ). - Table 2 gives an overview of the glenoid components implanted in this study.

The guide wire was then placed via a central sleeve. The glenoid reamer matching the previously determined glenoid size was inserted over the wire and any cartilage still present was removed, preserving as much subchondral bone as possible. The keel for the subsequent glenoid component was then prepared, rinsed, and dried. The keel and the backside of the glenoid component were then coated with high-viscosity pressurized polymethylmethacrylate cement and the glenoid component held in place with the compactor until the cement hardened. Following insertion of the humeral component, the subscapularis tendon was repaired with four to five nonresorbable sutures. The arm was immobilized postoperatively for 4 weeks on a shoulder abduction pillow at $15^{\circ}$ of abduction with passive exercise for 6 weeks followed by active exercise.

\section{Clinical follow-up}

Clinical follow-up was undertaken by two blinded observers (SS, JE) in the course of follow-up. The clinical parameters determined were:

- Range of motion on abduction (Abd)

- Flexion (Flex) and external rotation (Er)

- Constant Score (CS)

- Age- and sex-adapted Constant Score (CS\%)

- Subjective Shoulder Value (SSV)

- Objective satisfaction (very satisfied, satisfied, unsatisfied, very unsatisfied).

Complications and revision procedures were also recorded.

\section{Radiographic follow-up}

Radiographs of the affected shoulder in two planes (anteroposterior and axial) were obtained for all patients preoperatively, immediately postoperatively, and in the course of follow-up. In addition, magnetic resonance imaging (MRI) was performed preoperatively on all patients. Glenoid morphology was determined according to the method of Walch [35] as modified by Bercik et al. [3], together with glenoid version, based on the axial radiographs and MRI. Centering of the humeral head (in \%) was measured on
Table 2 Overview of the implanted

glenoid components in the two groups

Number $\boldsymbol{n}$ (\%)

\begin{tabular}{|l|l|}
\hline $\begin{array}{l}\text { Group } 1(n=42) \\
\text { Small }\end{array}$ & $1(2 \%)$ \\
\hline Medium & $21(50 \%)$ \\
\hline Large & $20(48 \%)$ \\
\hline Group $2(n=45)$ & \\
\hline Small $30 \mathrm{~mm}$ & $1(2 \%)$ \\
\hline Small $35 \mathrm{~mm}$ & $2(4 \%)$ \\
\hline Small $40 \mathrm{~mm}$ & $1(2 \%)$ \\
\hline Medium $30 \mathrm{~mm}$ & $5(11 \%)$ \\
\hline Medium $35 \mathrm{~mm}$ & $5(11 \%)$ \\
\hline Medium $40 \mathrm{~mm}$ & $7(16 \%)$ \\
\hline Large $40 \mathrm{~mm}$ & $16(36 \%)$ \\
\hline Large $50 \mathrm{~mm}$ & $5(11 \%)$ \\
\hline Large $60 \mathrm{~mm}$ & 0 \\
\hline Extra-large $40 \mathrm{~mm}$ & $2(4 \%)$ \\
\hline Extra-large $50 \mathrm{~mm}$ & $1(2 \%)$ \\
\hline Extra-large $60 \mathrm{~mm}$ & 0 \\
\hline
\end{tabular}

axial radiographs and centering was classified according to the method of Walch [35] based on the preoperative and postoperative axial radiographs. Determination of humeral centering based on the axial radiograph taken during follow-up was not possible in five patients.

On the immediately postoperative radiographs and the anteroposterior radiographs taken in the course of follow-up, the glenoid component was analyzed for the occurrence of radiolucent lines and loosening. Radiolucent lines around the glenoid were classified according to the method described by Molé [20]. The radiographic analysis was performed by two blinded observers (MS, SS) independently of one another. Agreement in respect of the assessment of radiolucent lines was analyzed statistically. In the event of discrepancies, a consensus was reached after discussion of the case.

\section{Statistical analysis}

Statistical analysis was performed with SPSS 23.0 software (IBM Corp., Ehningen, Germany). The level of significance was set at $p<0.05$. Differences between the two groups were calculated using the $t$ test for continuous data and scores and the chi-square test $(n \geq 5)$ or Fisher's exact test $(n<5)$ for categorical data. Interob- 
Obere Extremität 2021 · 16:68-74 https://doi.org/10.1007/s11678-020-00564-2

(c) The Author(s) 2020

\section{Schnetzke $\cdot$ S. Sulzer $\cdot$ J. Engelke $\cdot$ M. Loew}

\section{Cemented all-polyethylene glenoid with standard or individualized backside curvature. A retrospective comparative study}

\section{Abstract}

Background. The clinical and radiographic results of an all-polyethylene glenoid with variable backside curvature adapted to the individual anatomy were compared with a standard all-polyethylene glenoid. Methods. This retrospective study included 87 patients with primary glenohumeral osteoarthritis and anatomic shoulder replacement with a short-stem prosthesis and cemented all-polyethylene glenoid. In all, 42 patients with a mean age of $67 \pm 8$ years were treated with an all-polyethylene glenoid without variable curvature (group 1) and 45 patients with a mean age of $68 \pm 10$ years were treated with an all-polyethylene glenoid with individualized curvature (group 2).
Clinical outcome was determined by range of motion, Constant Score (CS) age- and sexadjusted Constant Score (CS\%), and Subjective Shoulder Value (SSV). Radiographs were analyzed for the occurrence of radiolucent lines according to the method of Mole. Results. After a mean follow-up of 31 months (range, 24-50) a significant improvement was achieved in all clinical parameters in both groups $(p<0.001)$. Immediately postoperatively, the radiolucent line score in group 1 was $0.8 \pm 1.4$ and in group 2 $0.7 \pm 1.1(p=0.713)$. At the final follow-up, the radiolucent line scores were $1.2 \pm 2.0$ in group 1 and $0.9 \pm 1.1$ in group $2(p=0.410)$. In five patients (12\%) from group 1 and four patients from group $2(9 \%)$, the radiolucent line score increased over time. One patient in group 1 (2\%) exhibited radiographic glenoid loosening.

Conclusion. The all-polyethylene glenoid with individualized curvature showed promising short-term clinical and radiographic results. Further studies with a longer follow-up are necessary to evaluate whether the new all-polyethylene glenoid is superior to the standard all-polyethylene glenoid.

\section{Keywords}

Radiolucent lines - Shoulder joint · Shoulder arthroplasty - Metal-backed. Shoulder replacement

\section{Vergleich eines zementierten individualisierten Polyethylen-Glenoids mit einem Standard- Polyethylen-Glenoid. Eine retrospektive Vergleichsstudie}

\section{Zusammenfassung}

Hintergrund. In dieser Studie wurden die klinischen und radiologischen Ergebnisse eines neuen Polyethylen-Glenoids mit variabler, an die individuelle Anatomie angepasster Kurvatur mit einem StandardPolyethylen-Glenoid verglichen.

Material und Methoden. In diese Studie wurden 87 Patienten mit primärer Omarthrose und anatomischem Schultergelenkersatz mit einer Kurzschaftprothese und zementiertem Polyethylen-Glenoid eingeschlossen. Davon wurden 42 Patienten (Durchschnittsalter $67 \pm 8$ Jahre) mit einem Polyethylen-Glenoid ohne variable Kurvatur (Gruppe 1) und $45 \mathrm{~Pa}$ tienten ( $68 \pm 10$ Jahre) mit einem PolyethylenGlenoid mit individualisierter Kurvatur (Gruppe 2) versorgt. Das klinische Ergebnis wurde anhand von Bewegungsumfang, Constant Score (CS), alters- und geschlechtsadaptiertem CS (CS\%) und subjektivem Schulterwert (SSV) erfasst. Anhand der Röntgenbilder wurde der Radiolucent-Score nach der Methode von Mole bestimmt.

Ergebnisse. Nach einer durchschnittlichen Follow-up-Dauer von 31 Monaten (24-50 Monate) wurde in beiden Gruppen eine signifikante Verbesserung der klinischen Parameter erzielt $(p<0,001)$. Der RadiolucentScore unmittelbar postoperativ betrug $0,8 \pm 1,4$ in Gruppe 1 und $0,7 \pm 1,1$ in Gruppe $2(p=0,713)$. Zum Zeitpunkt der Abschlussuntersuchung lag der RadiolucentScore bei 1,2 2,0 in Gruppe 1 und bei $0,9 \pm 1,1$ in Gruppe $2(p=0,410)$. Bei 5 von
42 Patienten (12\%) aus Gruppe 1 und 4 von 45 Patienten aus Gruppe 2 (9\%) nahm der Radiolucent-Score im Laufe der Zeit zu. Ein Patient in Gruppe 1 (2\%) zeigte radiologisch eine glenoidale Lockerung.

Schlussfolgerung. Das Polyethylen-Glenoid mit individualisierter Kurvatur weist gute kurzfristige klinische und radiologische Ergebnisse auf. Weitere Studien mit längerem Follow-up sind notwendig, um zu prüfen, ob das individualisierte Polyethylen-Glenoid dem Standard-Polyethylen-Glenoid überlegen ist.

\section{Schlüsselwörter}

Strahlendurchlässige Linien · Schultergelenk · Schulterprothese · Metallverstärkt · Schultergelenkersatz server variability for the determination of the radiolucent line score was calculated with Cohen's $\kappa$, and agreement strength was inferred in accordance with the recommendations of Landis and Koch [17].

\section{Results}

\section{Clinical results}

After an average follow-up period of 31 months (range, 24-50), a significant improvement was obtained in all clinical parameters. The CS improved from $29 \pm 9$ to $75 \pm 15(p<0.001)$ and CS\% from $39 \% \pm 12$ to $101 \% \pm 18(p<0.001)$. The SSV improved from $41 \pm 15$ to $88 \pm 14$ $(p<0.001)$. Pain was also significantly reduced at the latest follow-up (preoperative: $4.5 \pm 2.7$; postoperative: $13.6 \pm 2.0$; $p<0.001)$. A significant improvement was obtained in the range of motion for abduction (preoperative: $91^{\circ} \pm 36$; postoperative: $\left.148^{\circ} \pm 29 ; p<0.001\right)$, flexion (preoperative: $104^{\circ} \pm 32$; postoperative: $\left.150^{\circ} \pm 25 ; p<0.001\right)$, and external rotation (preoperative: $11^{\circ} \pm 19$; postoperative: $\left.45^{\circ} \pm 15 ; p<0.001\right)$. In all, 65 patients $(75 \%)$ were very satisfied with the clinical outcome at the time of follow-up, 20 patients (23\%) were satisfied, one patient $(1 \%)$ was unsatisfied, and one further patient ( $1 \%$ ) was very unsatisfied. The patients in group 1 reported a significantly better SSV preoperatively ( $44 \pm 14$ vs. $35 \pm 13 ; p=0.018$ ). The 
Table 3 Comparison of the preoperative clinical status of the two groups

\begin{tabular}{|c|c|c|c|}
\hline & Aequalis $(n=42)$ & Perform $(n=45)$ & $p^{a}$ \\
\hline Follow-up (months) & $30 \pm 5(24-40)$ & $32 \pm 10(24-50)$ & 0.247 \\
\hline CS pre-OP (pts) & $29 \pm 9$ & $28 \pm 8$ & 0.738 \\
\hline CS\% pre-OP & $39 \pm 12$ & $38 \pm 13$ & 0.711 \\
\hline SSV pre-OP (\%) & $44 \pm 14$ & $35 \pm 13$ & 0.018 \\
\hline Pain pre-OP (pts) & $4.5 \pm 2.4$ & $4.7 \pm 3.1$ & 0.746 \\
\hline Abd pre-OP $\left(^{\circ}\right)$ & $95 \pm 38$ & $85 \pm 33$ & 0.276 \\
\hline Flex pre-OP $\left({ }^{\circ}\right)$ & $106 \pm 33$ & $99 \pm 28$ & 0.353 \\
\hline ER pre-OP $\left(^{\circ}\right)$ & $13 \pm 21$ & $8 \pm 14$ & 0.301 \\
\hline
\end{tabular}

Table 4 Comparison of the clinical results of the two groups on follow-up

\begin{tabular}{ll|l|l} 
& Aequalis $(\boldsymbol{n}=\mathbf{4 2})$ & Perform $(\boldsymbol{n}=\mathbf{4 5})$ & $\boldsymbol{p}^{\mathbf{a}}$ \\
\hline CS (pts) & $75 \pm 13$ & $74 \pm 11$ & 0.657 \\
\hline CS\% & $102 \pm 19$ & $100 \pm 18$ & 0.615 \\
\hline SSV (\%) & $88 \pm 13$ & $88 \pm 15$ & 0.913 \\
\hline Pain (pts) & $13.4 \pm 2.1$ & $13.8 \pm 1.9$ & 0.296 \\
\hline Abd $\left(^{\circ}\right)$ & $150 \pm 28$ & $146 \pm 29$ & 0.525 \\
Flex $\left(^{\circ}\right)$ & $153 \pm 21$ & $148 \pm 28$ & 0.413 \\
ER $\left(^{\circ}\right)$ & $46 \pm 14$ & $44 \pm 15$ & 0.523
\end{tabular}

CS Constant Score, pts points CS\% age- and sex-adapted CS, SSV Subjective Shoulder Value, Abd abduction, Flex flexion, ER external rotation

${ }^{\mathrm{a}} \boldsymbol{t}$ Test

Table 5 Comparison of preoperative glenoid morphology and humeral centering between the two groups

\begin{tabular}{|c|c|c|c|}
\hline & Aequalis $(n=42)$ & Perform $(n=45)$ & $p$ \\
\hline \multicolumn{4}{|c|}{ Glenoid type (Walch) $(n ; \%)$} \\
\hline $\mathrm{A} 1$ & $1(2 \%)$ & 0 & \multirow[t]{6}{*}{$0.101^{\circ}$} \\
\hline $\mathrm{A} 2$ & $9(21 \%)$ & $15(33 \%)$ & \\
\hline B1 & $26(62 \%)$ & $18(40 \%)$ & \\
\hline B2 & $5(12 \%)$ & $12(27 \%)$ & \\
\hline B3 & 0 & 0 & \\
\hline C & $1(2 \%)$ & 0 & \\
\hline Glenoid retroversion $\left({ }^{\circ}\right)$ & $12.3 \pm 7.4$ & $10.3 \pm 11.4$ & $0.395^{\mathrm{b}}$ \\
\hline Humeral centering (\%) & $0.52 \pm 0.06$ & $0.54 \pm 0.06$ & $0.246^{b}$ \\
\hline \multicolumn{4}{|c|}{ Glenoid Index (Walch) ( $n$; \%) } \\
\hline Anterior & $3(7 \%)$ & $1(2 \%)$ & \multirow[t]{3}{*}{0.728} \\
\hline Centered & $28(67 \%)$ & $31(69 \%)$ & \\
\hline Posterior & $11(26 \%)$ & $13(29 \%)$ & \\
\hline
\end{tabular}

other parameters of preoperative status (- Table 3) and the clinical outcome at follow-up (-Table 4) were comparable $(p \geq 0.276)$.
(- Table 5). Preoperatively determined glenoid retroversion was $11.2^{\circ} \pm 9.8$. Humeral centering was $53 \% \pm 6$ preoperatively, and 59 shoulders (68\%) were classified as centered and 24 shoulders (28\%) as posteriorly decentered. There was no difference between the two groups regarding radiographic parameters preoperatively $(p \geq 0.101)$.

Interobserver agreement for the determination of the radiolucent line score was almost perfect $(\kappa=0.890)$. The radiolucent line score immediately postoperatively was $0.8 \pm 1.4$ in group 1 and $0.7 \pm 1.1$ in group $2(p=0.713)$. At the time of follow-up, the radiolucent line score was $1.2 \pm 2.0$ in group 1 and $0.9 \pm 1.1$ in group $2(p=0.421)$. In five of $42 \mathrm{pa}$ tients (12\%) in group 1 and four of 45 patients in group $2(9 \%)$ the radiolucent line score increased over time. The increase was not significant in group 1 $(p=0.344)$ and group $2(p=0.448)$. The radiolucent line score at the time of follow-up was more than 1 in 13 patients in group $1(31 \%)$ and in 10 patients in group $2(22 \%)(p=0.356)$. Detailed analysis showed that radiolucent lines occurred most frequently in zones 3 and 4 in both groups, although in group 2 the same number or fewer radiolucent lines were present in all zones (• Fig. 1).

Glenoid loosening occurred at the time of follow-up in one of 87 patients (1\%) with a radiolucent line score of 10 (- Fig. 2).

As the clinical outcome is still satisfactory (CS 71, SSV 75, pain 13), the patient has refused surgical revision to date. The detailed radiographic results are summarized in • Table 6 .

Complications occurred in two patients ( $2 \%$; both group 1$)$. In addition to the previously described glenoid loosening, one patient had to undergo revision for a postoperative hematoma.

\section{Discussion}

Anatomic shoulder replacement is a safe and effective method of treating primary glenohumeral osteoarthritis and other shoulder joint disorders [26]. Owing to greater life expectancy combined with a high functional requirement, the number of primary and revision procedures 


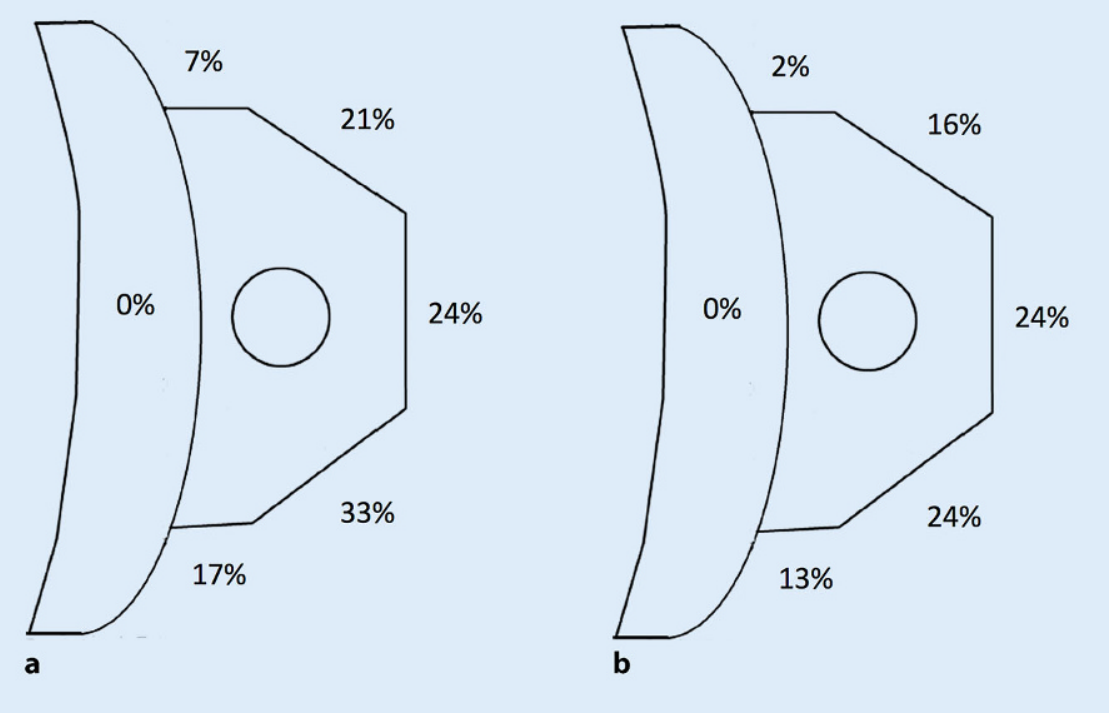

Fig. $1 \Delta$ Overview of radiolucent lines in group 1 (a) and group 2 (b) showing localization and frequency at the time of follow-up. (Modified from [27])
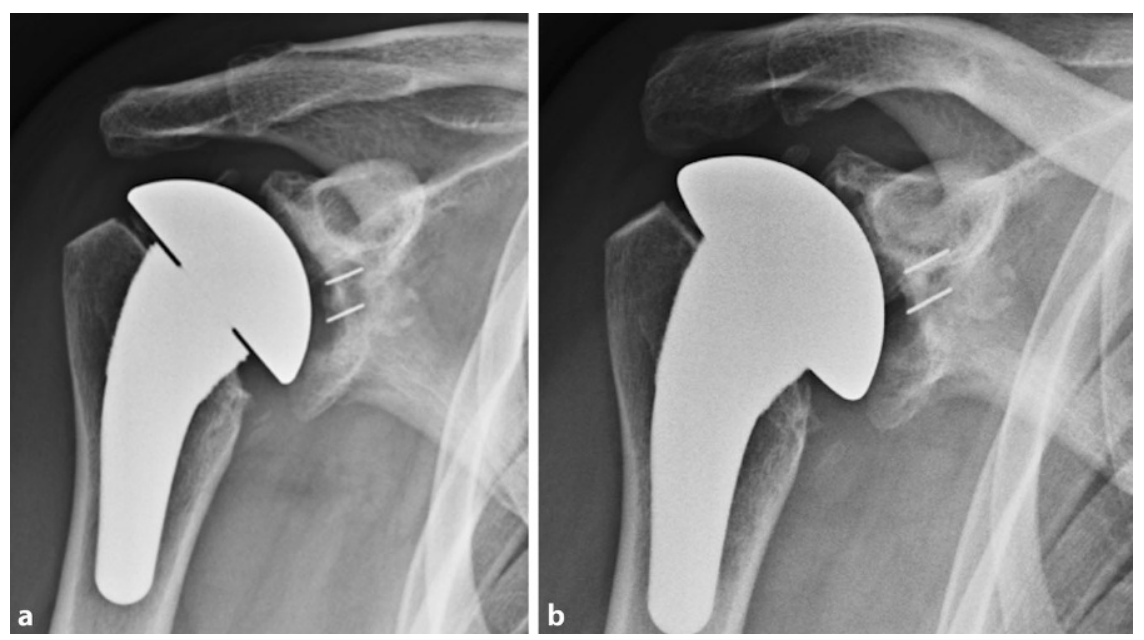

Fig. 2 × Radiographic evidence of glenoid loosening. a Radiolucent lines in zones 1, 2, and 3 already present after 2 years. $\mathbf{b}$ Increase in radiolucent lines around the whole glenoid component after 5 years

will increase further in the future [25]. Various systems of stemless or short-stem prostheses have been successfully introduced onto the market in recent years to simplify revision procedures [31]. However, the weak link of anatomic shoulder replacement is not the stem but the glenoid component. In a prospective observational study, Raiss et al. showed that glenoid loosening occurs, in particular, in the long term [32]. A patient cohort had a radiolucent line score 7 years postoperatively of 1.8 , but there was a significant increase to 8.2 points after 13 years. These data show that glenoid loosening constitutes a relevant clinical problem.
The anatomy of the glenoid is highly variable, and insufficient allowance is made for this in traditional glenoid components with only one radius of curvature of the backside [36]. Because of the variable anatomy of the glenoid, (almost) complete removal of subchondral bone is required in many cases in order to adapt the anatomy of the glenoid to the existing design of the glenoid component. However, preparation of the glenoid with the removal of subchondral bone has been identified as a substantial risk factor for the development of glenoid loosening [37]. This was the rationale for the development of new glenoid im- plants with variable backside curvature adapted to the individual anatomy.

In this study, the clinical and radiographic results of a new all-polyethylene glenoid with variable backside curvature adapted to the individual anatomy were compared with a standard all-polyethylene glenoid.

A significant improvement in all clinical parameters was obtained in both groups with no differences between the two groups. The results from the present study are comparable to the previously published results following total shoulder arthroplasty with third- and fourthgeneration prostheses.

The radiographic results of the present study are promising with a low rate of radiolucent lines in the 31-month followup period. The radiolucent line scores in both groups were low with no differences between the two groups. The hypothesis postulated initially must therefore be rejected. Detailed analysis shows that the new all-polyethylene glenoid with variable backside curvature adapted to the individual anatomy exhibits the same number or fewer radiolucent lines in all six zones. Furthermore, analysis showed that a smaller proportion of cases $(22 \%$ vs. $33 \%$ ) had more than one radiolucent line.

The results of the current study confirm those of Raiss at al., who recently published the outcomes of 118 cases with a follow-up period of 38 months using the same glenoid component [27]. They reported that the mean radiolucent line score after 38 months was 1.06 points, which is comparable to the results of the current study. Radiographic glenoid loosening was not observed in their study.

Compared with previously published studies of radiographic outcomes with a comparable or pegged design or only partially cemented components, the rate of radiolucent lines in this study is very low $[10,12,19,24,40]$. Kasten et al. in 2010 analyzed the radiographic outcomes of 96 cases treated with a cemented all-polyethylene glenoid with a standard backside and showed radiographically that glenoid loosening had increased from $9 \%$ after 5 years to $33 \%$ after 9 years [15]. By adapting the glenoid component to the individual anatomy 
Table 6 Comparison of the radiographic results at the time of follow-up between the two groups

\begin{tabular}{|c|c|c|c|}
\hline & Aequalis $(n=42)$ & Perform $(n=45)$ & $p$ \\
\hline RLL score, postoperative & $0.8 \pm 1.4$ & $0.7 \pm 1.1$ & $0.713^{b}$ \\
\hline RLL score, final follow-up & $1.2 \pm 2.0$ & $0.9 \pm 1.1$ & $0.421^{\mathrm{b}}$ \\
\hline RLL score > $1(n ; \%)$ & $13(31 \%)$ & $10(22 \%)$ & $0.356^{\mathrm{a}}$ \\
\hline Glenoid loosening $(n ; \%)$ & $1(2 \%)$ & 0 & $0.483^{c}$ \\
\hline Glenoid retroversion $\left({ }^{\circ}\right.$ ) & $10.4 \pm 8.5$ & $9.1 \pm 6.0$ & $0.448^{b}$ \\
\hline Humeral centering (\%) & $0.45 \pm 0.06$ & $0.47 \pm 0.05$ & $0.093^{b}$ \\
\hline \multicolumn{4}{|c|}{ Glenoid Index (Walch) ( $n$; \%) } \\
\hline Anterior & $22(52 \%)$ & $14(31 \%)$ & \multirow[t]{4}{*}{$0.197^{\circ}$} \\
\hline Centered & $18(43 \%)$ & $25(60 \%)$ & \\
\hline Posterior & $1(2 \%)$ & $2(4 \%)$ & \\
\hline NA & $1(2 \%)$ & $4(9 \%)$ & \\
\hline \multicolumn{4}{|c|}{$\begin{array}{l}\text { NA not available, } R L L \text { radiolucent line } \\
{ }^{2} \text { Chi-square test } \\
{ }^{\mathrm{b}} \boldsymbol{t} \text { Test } \\
\text { 'Fisher's exact test }\end{array}$} \\
\hline
\end{tabular}

of the shoulder, it is hoped to achieve a long-term reduction in the rate of glenoid loosening.

Alternative designs of the glenoid component include the pegged allpolyethylene glenoid and the metalbacked glenoid. The pegged all-polyethylene glenoid shows good radiographic results in the medium-term followup period [9, 11, 18]. One cause for concern, however, is the "ballooning" phenomenon of osteolysis around the central peg, which is described in more than $50 \%$ of cases $[2,21]$. Metal-backed glenoids have the great advantage over conventional all-polyethylene glenoids of convertibility, although many metalbacked glenoids exhibit high rates of osteolysis within the first few years and should therefore be used with some degree of reservation [6, 14, 22, 39]. Watson et al. have recently published 2-year results following implantation of a trabecular metal-backed glenoid in anatomic total shoulder arthroplasty and found $25 \%$ rates of radiographic metal debris and osteolysis at a minimum 2-year follow-up in this series with one catastrophic failure [39]. The authors concluded that this implant should be used with caution, and patients must be followed up closely. As far back as 2002, Boileau et al. published the results of a prospectively randomized study comparing a cemented polyethylene component with uncemented metal- backed glenoid components. After a follow-up period of 36 months, three of 20 patients had to undergo revision because of loosening of the metal-backed glenoid. At the same time, however, the rate of radiolucent lines was significantly higher with the polyethylene glenoid than with the metal-backed glenoid ( $85 \%$ vs. 25\%). The authors therefore concluded that efforts must continue to improve glenoid component design and fixation [5].

In summary, the best possible evidence from long-term data is currently available for the cemented keeled glenoid component. However, even with cemented keeled all-polyethylene glenoids, high loosening rates have been observed during long-term follow-up. The design of existing all-polyethylene glenoids has been adapted and now, instead of a standard backside, backsides with variable curvature adapted to the individual anatomy are available. The aim of these is to adapt the glenoid components to the variable anatomy of the native glenoid and not vice versa. This study confirms the promising results of Raiss et al. [27], but further studies with a longer follow-up are necessary for a definitive assessment.

\section{Limitations}

This study has several limitations. The study design is retrospective, and the study hypothesis was postulated retrospectively. Furthermore, the patients were not randomized to the surgical procedure. A further limitation is the short follow-up period. The strengths of the study lie in the standard surgical and follow-up treatment procedures, since the same surgeon treated all patients. A further strength of the study is that two highly homogeneous patient groups with respect to age, sex, and diagnosis, and differing only in the design of the glenoid component, were compared.

\section{Practical conclusion}

- The results of the new all-polyethylene glenoid with variable backside curvature adapted to the individual anatomy are promising.

- At short-term follow-up, the standard all-polyethylene glenoid and the new all-polyethylene glenoid with variable backside curvature do not show significant differences.

- Further studies with a longer-term follow-up are required to investigate whether the new design has a positive effect on outcome

\section{Corresponding address}

PD Dr. Marc Schnetzke
BG Trauma Center
Ludwigshafen, University of
Heidelberg
Ludwig-Guttmann-Straße 13,
67071 Ludwigshafen,
Germany
marc.schnetzke@atos.de

Funding. Open Access funding provided by Projekt DEAL.

\section{Compliance with ethical guidelines}

Conflict of interest. M. Schnetzke, S. Sulzer, and J. Engelke, their immediate families, and any research foundations with which they are affiliated have not received any financial payments or other benefits from any commercial entity related to the subject of this article. M. Loew received royalties from Tornier/ Wright, which is related to the subject of this work. No company had any input into the study design, protocol, testing, data analysis, or manuscript preparation.

All procedures performed in studies involving human participants were in accordance with the ethical stan- 
dards of the institutional and/or national research committee and with the 1964 Helsinki declaration and its later amendments or comparable ethical standards (ethical committee of Heidelberg University, application number 6/16). Informed consent was obtained from all individual participants included in the study.

Open Access. This article is licensed under a Creative Commons Attribution 4.0 International License, which permits use, sharing, adaptation, distribution and reproduction in any medium or format, as long as you give appropriate credit to the original author(s) and the source, provide a link to the Creative Commons licence, and indicate if changes were made. The images or other third party material in this article are included in the article's Creative Commons licence, unless indicated otherwise in a credit line to the material. If material is not included in the article's Creative Commons licence and your intended use is not permitted by statutory regulation or exceeds the permitted use, you will need to obtain permission directly from the copyright holder. To view a copy of this licence, visit http://creativecommons.org/licenses/by/4.0/.

\section{References}

1. Aldinger PR, Raiss P, Rickert $M$ et al (2010) Complications in shoulder arthroplasty: an analysis of 485 cases. Int Orthop 34:517-524

2. Arnold RM, High RR, Grosshans KT et al (2011) Bone presence between the central peg's radial fins of a partially cemented pegged all poly glenoid component suggest few radiolucencies. JShoulder Elbow Surg 20:315-321

3. Bercik MJ, Kruse K 2nd, Yalizis M et al (2016) A modification to the Walch classification of the glenoid in primary glenohumeral osteoarthritis using three-dimensional imaging. J Shoulder Elbow Surg 25:1601-1606

4. Bohsali KI, Wirth MA, Rockwood CA Jr. (2006) Complications of total shoulder arthroplasty. JBone Joint Surg Am 88:2279-2292

5. Boileau P, Avidor C, Krishnan SG et al (2002) Cemented polyethylene versus uncemented metal-backed glenoid components in total shoulder arthroplasty: a prospective, doubleblind, randomized study. J Shoulder Elbow Surg 11:351-359

6. Boileau P, Moineau G, Morin-Salvo N et al (2015) Metal-backed glenoid implant with polyethylene insert is not a viable long-term therapeutic option. JShoulderElbow Surg 24:1534-1543

7. Collin P, Tay AK, Melis B et al (2011) A ten-year radiologic comparison of two-all polyethylene glenoid component designs: a prospective trial. JShoulder Elbow Surg 20:1217-1223

8. Deshmukh AV, Koris M, Zurakowski D et al (2005) Total shoulder arthroplasty: long-term survivorship, functional outcome, and quality of life. JShoulder Elbow Surg 14:471-479

9. Edwards TB, Labriola JE, Stanley RJ et al (2010) Radiographic comparison of pegged and keeled glenoid components using modern cementing techniques: a prospective randomized study. JShoulder Elbow Surg 19:251-257

10. Fox TJ, Cil A, Sperling JW et al (2009) Survival of the glenoid component in shoulder arthroplasty. JShoulder Elbow Surg 18:859-863

11. Gartsman GM, ElkousyHA, Warnock KMetal (2005) Radiographic comparison of pegged and keeled glenoid components. J Shoulder Elbow Surg 14:252-257
12. Greiner S, Berth A, Kaab M et al (2013) Glenoid morphology affects the incidence of radiolucent lines around cemented pegged polyethylene glenoid components. Arch Orthop Trauma Surg 133:1331-1339

13. Habermeyer P, Lichtenberg S, Tauber M et al (2015) Midterm results of stemless shoulder arthroplasty: a prospective study. J Shoulder Elbow Surg 24:1463-1472

14. Hsu JE, Hackett DJ Jr., Vo KV et al (2018) What can be learned from an analysis of 215 glenoid component failures? J Shoulder Elbow Surg 27:478-486

15. Kasten P, Pape G, Raiss P et al (2010) Mid-term survivorship analysis of a shoulder replacement with a keeled glenoid and a modern cementing technique. J Bone Joint Surg Br 92:387-392

16. Khan A, Bunker TD, Kitson JB (2009) Clinical and radiological follow-up of the Aequalis thirdgeneration cemented total shoulder replacement: a minimum ten-year study. J Bone Joint Surg 91:1594-1600

17. Landis JR, Koch GG (1977) The measurement of observer agreement for categorical data. Biometrics 33:159-174

18. Lazarus MD, Jensen KL, Southworth C et al (2002) The radiographic evaluation of keeled and pegged glenoid component insertion. J Bone Joint Surg Am 84-a:1174-1182

19. Merolla G, Ciaramella G, Fabbri E et al (2016) Total shoulder replacement using a bone ingrowth central peg polyethylene glenoid component: a prospective clinical and computed tomography study with short- to mid-term follow-up. Int Orthop 40:2355-2363

20. Mole DR, Riand N, Levigne C, Walch G (1999) Cemented glenoid components: results in osteoarthritis and rheumatoid arthritis. Springer Berlin Heidelberg

21. Noyes MP, Meccia B, Spencer EE Jr. (2015) Five- to ten-year follow-up with a partially cemented allpolyethylene bone-ingrowth glenoid component. JShoulder Elbow Surg 24:1458-1462

22. Papadonikolakis A, Matsen FA 3rd (2014) Metalbacked glenoid components have a higher rate of failure and fail by different modes in comparison with all-polyethylene components: a systematic review. JBone Joint Surg Am 96:1041-1047

23. Papadonikolakis A, Neradilek MB, Matsen FA 3rd (2013) Failure of the glenoid component in anatomic total shoulder arthroplasty: a systematic review of the English-language literature between 2006 and 2012. J Bone Joint Surg 95:2205-2212

24. Parks DL, Casagrande DJ, Schrumpf MA et al (2016) Radiographic and clinical outcomes of tota shoulder arthroplasty with an all-polyethylene pegged bone ingrowth glenoid component: prospective short- to medium-term follow-up. JShoulder Elbow Surg 25:246-255

25. Raiss P, Aldinger PR, Kasten P et al (2008) Total shoulder replacement in young and middle-aged patients with glenohumeral osteoarthritis. JBone Joint Surg Br 90:764-769

26. Raiss P, Bruckner T, Rickert $M$ et al (2014) Longitudinal observational study of total shoulder replacements with cement: fifteen to twenty-year follow-up. J Bone Joint Surg Am 96:198-205

27. Raiss P, Godeneche A, Wittmann T et al (2018) Short-term radiographic results of a cemented polyethylene keeled glenoid component with varying backside radiuses of curvature. J Shoulder Elbow Surg 27:839-845

28. Raiss P, Schmitt M, Bruckner T et al (2012) Results of cemented total shoulder replacement with a minimum follow-up of ten years. J Bone Joint Surg Am 94:e1711-e1710
29. Schnetzke M, Coda S, Raiss P et al (2016) Radiologic bone adaptations on a cementless short-stem shoulder prosthesis. J Shoulder Elbow Surg 25:650-657

30. Schnetzke M, Loew M, Raiss P et al (2019) Shortstem anatomical shoulder replacement-a systematic review. Obere Extrem 14:139-148

31. Schnetzke M, Rick S, Raiss P et al (2018) Mid-term results of anatomical total shoulder arthroplasty for primary osteoarthritis using a short-stemmed cementless humeral component. Bone Joint J 100b:603-609

32. Sowa B, Bochenek M, Bulhoff $M$ et al (2017) The medium- and long-term outcome of total shoulder arthroplasty for primary glenohumeral osteoarthritis in middle-aged patients. Bone Joint J 99-b:939-943

33. Stewart MP, Kelly IG (1997) Total shoulder replacement in rheumatoid disease: 7- to 13year follow-up of 37 joints. J Bone Joint Surg $\mathrm{Br}$ 79:68-72

34. Verborgt O, El-Abiad R, Gazielly DF (2007) Longterm results of uncemented humeral components in shoulder arthroplasty. J Shoulder Elbow Surg 16:S13-S18

35. Walch G, Badet R, Boulahia A et al (1999) Morphologic study of the glenoid in primary glenohumeral osteoarthritis. J Arthroplasty 14:756-760

36. Walch G, Mesiha M, Boileau P et al (2013) Threedimensional assessment of the dimensions of the osteoarthritic glenoid. Bone Joint J 95b:1377-1382

37. Walch G, Young AA, Boileau P et al (2012) Patterns of loosening of polyethylene keeled glenoid components after shoulder arthroplasty for primary osteoarthritis: results of a multicenter study with more than five years of follow-up. JBone Joint Surg Am 94:145-150

38. Walch G, Young AA, Melis B et al (2011) Results of a convex-back cemented keeled glenoid component in primary osteoarthritis: multicenter study with a follow-up greater than 5 years. JShoulderElbow Surg 20:385-394

39. Watson ST, Gudger GK Jr., Long CD et al (2018) Outcomes of trabecular metal-backed glenoid components in anatomic total shoulder arthroplasty. JShoulder Elbow Surg 27:493-498

40. Wijeratna M, Taylor DM, Lee S et al (2016) Clinical and radiographic results of an all-polyethylene pegged Bone-Ingrowth Glenoid component. JBone Joint Surg Am 98:1090-1096

41. Young A, Walch G, Boileau P et al (2011) A multicentre study of the long-term results of using a flat-back polyethylene glenoid component in shoulder replacement for primary osteoarthritis. JBone Joint Surg Br 93:210-216 